\title{
A Good Long-Term Outcome of Early Telemedicine for a SARS-CoV-2 Patient in Wuhan China
}

\author{
Wei Chen ${ }^{1,2 *}$, Victoria J Wang ${ }^{3}$, Zhibin Zhang ${ }^{4}$, Ruizhen $\mathrm{Xu}^{5}$, Jinjun Qiu ${ }^{6}$, Guoping Sun ${ }^{1}$, and Shi V Liu ${ }^{7 *}$ \\ ${ }^{1}$ Department of Radiology, Pingshan District People's Hospital, Pingshan General Hospital of Southern Medical University, Shenzhen, China \\ ${ }^{2}$ Department of Radiology, Tongji Hospital, Tongji University School of Medicine, Shanghai, China \\ ${ }^{3}$ Department of Nephrology, Tufts Medical Center, Boston, USA \\ ${ }^{4}$ Department of Neurosurgery, Pingshan District People's Hospital, Pingshan General Hospital of Southern Medical University, Shenzhen, \\ China \\ ${ }^{5}$ Outpatient Department, Pingshan District People's Hospital, Pingshan General Hospital of Southern Medical University, Shenzhen, China \\ ${ }^{6}$ Department of Community Health, Pingshan District People's Hospital, Pingshan General Hospital of Southern Medical University,
} Shenzhen, China

${ }^{7}$ Eagle Institute of Molecular Medicine, Apex, NC, USA

*Corresponding author: Dr. Wei Chen, Department of Radiology, Pingshan District People's Hospital, Pingshan General Hospital of Southern Medical University, Shenzhen, Guangdong, 518118, China, Email; 1530831809@qq.com;

Dr. Shi Liu, Eagle Institute of Molecular Medicine, Apex, NC, USA, Email: svl8epa@gmail.com

Received: 弊 December 07, 2020

Published: 蹧 December 16, 2020

\begin{abstract}
Background: The coronavirus disease 2019(COVID-19) still continues its threat to more and more people in even wider area. Many challenges remain as to how to efficiently manage the health risk and effectively reduce the social disturbance of this ongoing pandemic.

Methods: A 45-year-old COVID-19 woman living in Wuhan China, the initial epicenter of this global pandemic, isolate-at-home by herself, was given an early individual-based, situation and disease condition, as well as symptom-specific telemedicine.

Results: The patients who had abnormal pulmonary CT findings was effectively treated with timely telemedicine composed of multiple approaches. Up to now, the patient feels normal on all aspects and her family members have been remained infection-free as well.

Conclusions: The low dose of exposure and the early proper treatment and rehabilitation might helped overcome the disease without any lasting damage. Primary care physicians who knew their patients very well can work with radiologists and other specialists in a telemedicine mode to provide a safe, trusted, high quality professional health care and a cost-effective, situation-and disease condition, as well as symptom-specific medical treatment for SARS-CoV-2 infection.
\end{abstract}

Keywords: SARS-CoV-2 , COVID-19, Telemedicine, Integrative medicine, Rehabilitation, Exposure control, Digital Record

The infection by severe acute respiratory syndrome coronavirus 2 (SARS-CoV-2) has now become a global pandemic with over 27486960 confirmed cases and resulted in 894983 deaths [1] since its first report in January 2020 [2]. World-wide efforts are being made in finding better ways for controlling the infection and treating the disease formally called as COVID-19 (Coronavirus Disease 2019)
[3]. However, many challenges remain as to how to efficiently manage the health risk and effectively reduce the social disturbance of this ongoing pandemic. We would like to share our experience in successfully treating a patient infected with SARS-CoV-2 in the early time of the outbreak of this disease in Wuhan, China though telemedicine while the patient was self-isolated at home. We believe that this mode of medical treatment of SARS-CoV-2 may play a more 
important role in future for efficient containment of the infection and for convenient treatment of the patients.

Our patient is a 45-year-old woman living in Wuhan China, the initial epicenter of this global pandemic. She did not have underlying health problems but routinely wore facial mask during the peak time of SARS-CoV-2 infection outbreak in Wuhan because she had a minor facial cosmetic surgery. She took off facial mask in the public place only once when she attended a dinner on Jan. 20, 2000 with two of her close girlfriends in a small restaurant. She normally took vitamin $\mathrm{C}$ and zinc supplement on daily basis. As a precaution, she also took moxifloxacin hydrochloride tablets from January 18 to 23, 2020 before she felt any symptom.

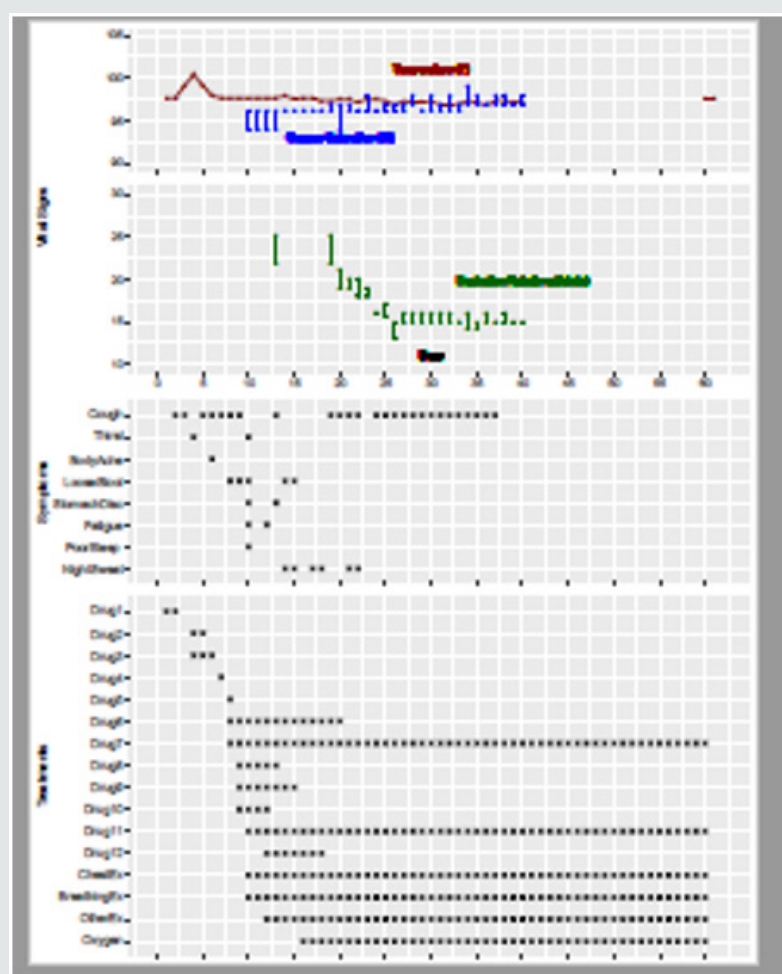

Figure 1: Time course of disease and treatment.

Patient's vital signs such as body temperature, blood oxygen saturation and respiration rate and symptoms such as cough, body-ache, fatigue etc. are plotted along with the various treatments which include taking medicines (see details listed below), breathing in oxygen, and performing chest and breathing exercise.

Drug 1: EVE quick pain killer; drug 2: cold capsule; drug 3: ofloxacin; drug 4: cephalosporin; drug 5: moxifloxacin hydrochloride iv; drug 6: arbidol hydrochloride; drug 7: Chinese medicine "LiKeJun" for raising white blood cell counts; drug 8: Chinese medicine "Lianhuaqingwen exerts" claimed of having anti-viral effects and reducing cytokine secretion; drug 9: human immune globin; drug 10: moxifloxacin hydrochloride tablet; drug 11: nutritional and supplementary treatments; drug 12: azithromycin.

Note: The patient felt just headache on day 1 and took an over-the -counter (OTC) "pain killer". She had occasional dry cough from day 2 and low fever from day 3 for which she took an OTC "cold capsule", and ofloxacin, from day 4 to day 6 . On day 7 she took cephalosporin tablet. We instructed the patient took arbidol hydrochloride tablet and "LiKeJun" from day 8 to day 20. Meanwhile, from day 8 to day 11, moxifloxacin hydrochloride tablets were taken again because our patients complained myalgia, fatigue, stomach pain and loose bowel movement. These medicines were replaced with azithromycin between day 12 and 18. Human immune globin was given between day 9 and 14. Beginning on day 1 our patient was able to self-monitor her blood oxygen level which ranged between 93 and $96 \%$. Her blood oxygen level was prevented from going down when a portable oxygen generator was purchased and placed into use on day 10. She breathed in oxygen three times a day for 2-3 min each time. Then she did chest and respiratory exercise according to our direction after each oxygen treatment.

The patient began to feel headache on January 24, 2020 which was the Eve of Chinese New Year. Two days later she began to have low fever and some dry cough. Because Wuhan was already placed under a lockdown on January 23 and normal medical treatment was extremely difficult to seek, the patient decided to isolate-athome by herself and found a way to obtain telemedicine from us for her treatment. Considering the outbreak of SARS-CoV-2 infection in Wuhan we speculated that our patient might be a victim of this virus' attack. Since we also believed that COVID-19 was more like a repeated outbreak of SARS [4] we thus borrowed many experiences from past SARS treatment. We closely monitored our patient's symptoms and signs, carefully analyzed her lab tests and imaging 
results, and gave situation-and symptom-specific treatment. We coded and quantified them in a figure for the convenient observation (Figure 1). The patient was formally diagnosed with a pneumonia on day 8 January 31, 2020 when her CT image showed a pneumonia sign with an obvious patchy consolidation shadows of exudative lesions in the left inferior lobe of her lung, and her blood test showed the total number of leukocytes decreased with lowered lymphocyte and neutrophil count and increased monocytes percentage, as well as C-reactive protein and hypersensitive c-reactive protein increased. Therefore, on day 8 she was given moxifloxacin hydrochloride via iv and arbidol hydrochloride tablets, as well as Leucogen Tablets which has been claimed with an effect of raising white blood cell counts [5] and whose Chinese name was called "LiKe Jun" (Figure 1). Due to limitations resulted from lockdown of Wuhan, our patient could took only four laboratory tests on day 8,11,12and 14 and three CT examinations on day 8, 14 and 61 over the course of the treatment (Figure 2). On day 11, her hypersensitive c-reactive protein still increased, but her C-reactive protein was back to the normal. On day 12 , her lymphocyte count increased and reached to the bottom line of the normal range, her total number of leukocytes also increased, but still lower than the bottom line of the normal range, meanwhile, her hypersensitive c-reactive protein still increased. On day 14, her blood test showed lymphocyte and leukocytes count increased than the ones on day 12 , and total number of leukocytes was back to bottom line of the normal range, her hypersensitive c-reactive protein decreased to the normal range, however, the percentage of lymphocyte count still lower than the bottom line of the normal range on day14, her CT still showed a pneumonia sign with less inflammation than the one on day 8. We evaluated patient's lab test results and made instant adjustments of our treatment. From day 9 we added another Chinese medicine called "Lianhuaqingwen exerts" and kept it in the treatment regime until day 14 . We selected this particular Chinese medicine because of its known antiviral effect from the traditional Chinese medicine [6]. At the time of our writing this manuscript, we learnt that a very recent publication even showed its anti-viral effect directly on SARS-CoV-2 and showed suppression of cytokine secretion by SARS-CoV-2 infected cells [7]. After all the above treatments and the recovery from illness, our patient found an opportunity to test her blood for antibody against SARS-CoV-2 on March 24, 2020. Her blood test showed presence of IgG against SARS-CoV-2, indicating that she was indeed infected with SARSCoV-2.

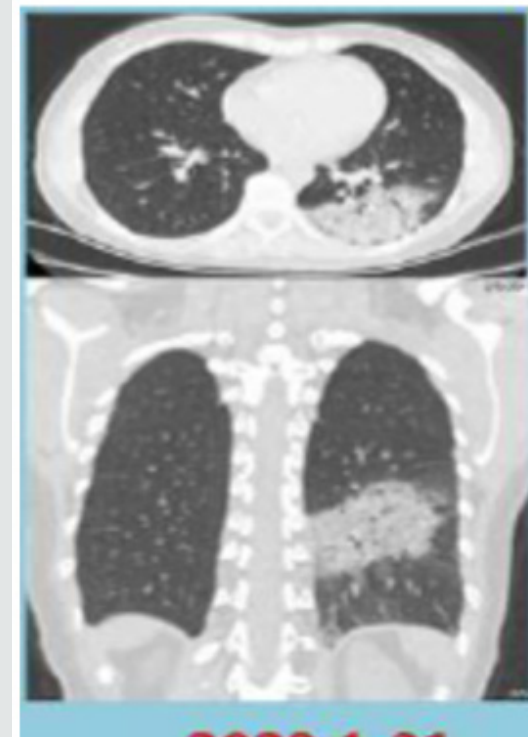

2020-1-31

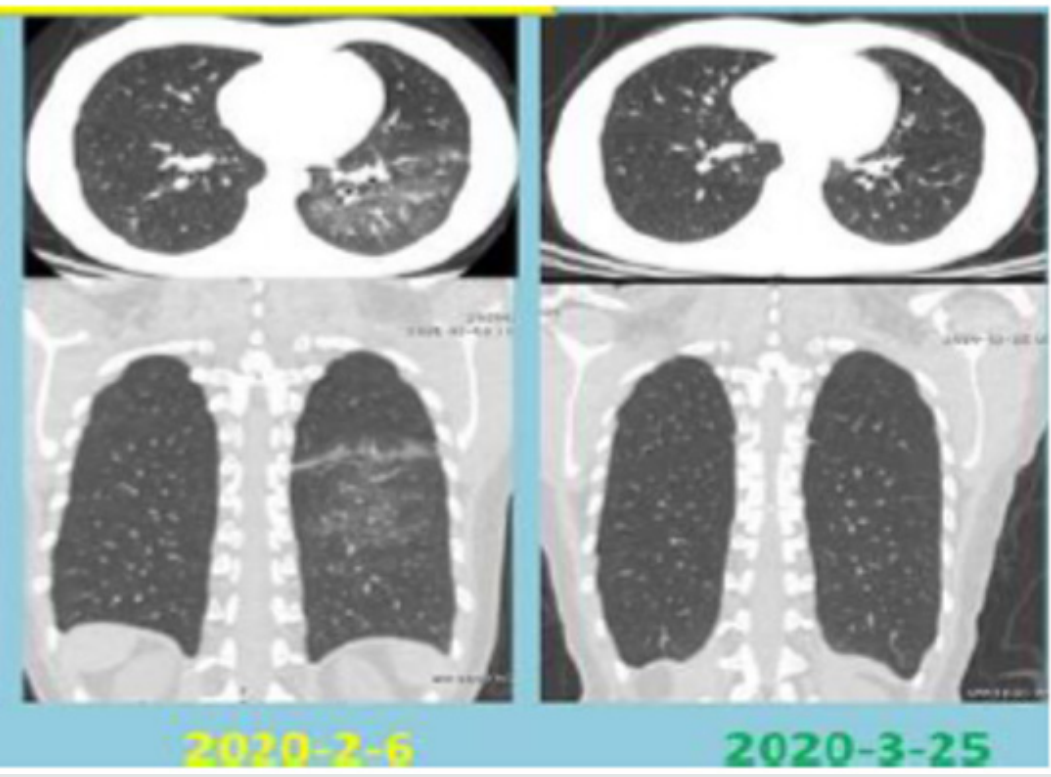

2020-3-25

Figure 2: CT images showing patient's progressive lung recovery from infection damages.

We witnessed the improvement of our patients' symptoms following our treatment. Her second CT image done on day 14 of February 6, 2020 began to show a great improvement. Her third CT examination on March 25, 2020 confirmed complete recovery from earlier lung damage $61^{\text {th }}$ day post her illness.

We maintained contact with our patient even up to now. The patient feels normal on all aspects and her family members have been remained infection-free as well. Thus, we had effectively treated a patient suffering a pneumonia caused by SARS-CoV-2 infection via telemedicine. We believe that our success was mainly contributed by our individual-based and situation-adjusted treatment regime that matches well with patient's health status and catches up with dynamic disease progression.

We realized that pulmonary inflammation might be a great contributor for severe acute respiratory syndrome (SARS) and thus paid special attention in utilizing a combination of medicines 
for suppressing this pathogenesis and for preventing potential cytokine storm. These measures might have helped the patient from slipping into severe stages that are difficult to treat even with advanced medical equipment and techniques in hospital-settings. We also feel that a most important point in managing SARS-CoV-2 infection is limiting the exposure to the virus.

Our patient was unlucky to be infected when she took off her facial mast to have a dinner with her close friends as one of them was suffering COVID -19 without obvious symptoms. The low dose of exposure and the early proper treatment and physical rehabilitation might help the better cure. One thing that may be worthy of mention is that our patient took vitamin $\mathrm{C}$ and zinc routinely. Some studies suggest that zinc may be useful for prophylaxis and treatment of COVID-19 [8].

As the COVID-19 pandemic is still continuing its threat to more and more people in even wider area, we believe that telemedicine should become an important route for safe and cost-effective rendering of patient's situation-specific treatment. We strongly believe that primary care physicians who knew their patients very well can work with radiologists and other specialist in a telemedicine mode to provide a safe, trusted, high quality professional health care and a cost-effective, situation-and disease condition, as well as symptom-specific medical treatment for SARS-CoV-2 infection, which promotes the integrated care and rehabilitation medicine in the face of acute infectious disease. Meanwhile, the integrated digital recording and displaying of medical histories, including patient's symptoms, vital signs, as well as others aspects such as treatment approaches may shed some light on the future medical records augmented with some new techniques. This digital medical history recoding of patients doesn't only facilitate practice of telemedicine, but also promotes exchanges of information and consolidation of big data. It provides a basis for introducing machine learning (ML) and artificial intelligence (AI) to play a big role in global efforts on fighting a daunting pandemic which threatens human lives around the world and causes massive social disturbance in human societies.

\section{References}

1. WHO Coronavirus Disease (COVID-19) Dashboard.

2. Zhu N, Zhang D, Wang W, Xingwang Li, Yang Bo, et al. (2019) A novel coronavirus from patients with pneumonia in China. NEJM 382(8): 727 733.

3. Wiersinga WJ, Rhodes A, Cheng AC, Peacock SJ, Prescott HC, et al. (2019) Pathophysiology,transmission,diagnosis, and treatment of Coronavirus Disease 2019 (COVID-19). JAMA 324(8): 782-793.

4. Sun Z, Thilakavathy K, Kumar SS, He G, Liu SV, et al. (2020) Potential factors influencing repeated SARS outbreaks in China. Int J Environ Res Pub Health Health 17(5): 1633.

5. Jiang SQ, Sun HX, Xu YM, Jiang YL, Pei JW, et al. (2011) Effects of jingyuankang capsules on leukocyte level in AIDS patients. J Tradit Chin Med 31(1): 32-35.

6. Runfeng L, Yunlong H, Jicheng H, Weiqi P, Qinhai M, et al. (2020) Lianhuaqingwen exerts anti-viral and anti-inflammatory activity against novel coronavirus (SARS-CoV-2), Pharmacological Research 156: 104761.

7. Ding Y, Lijuan Zeng, Runfeng Li, Qiaoyan Chen, Beixian Zhou et al. (2017) The Chinese prescription lianhuaqingwen capsule exerts anti-influenza activity through the inhibition of viral propagation and impacts immunefunction, BMC Complement. Altern Med 17(1): p. 130.

8. Kumar A, Kubota Y, Chernov M, Kasuya H (2020) Potential role of zinc supplementation in prophylaxix and treatment of COVID-19. Med Hypotheses 144: 109848.

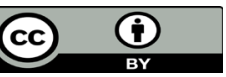

This work is licensed under Creative Commons Attribution 4.0 License

To Submit Your Article Click Here: Submit Article
DOI: $10.32474 /$ OAJOM.2020.04.000183

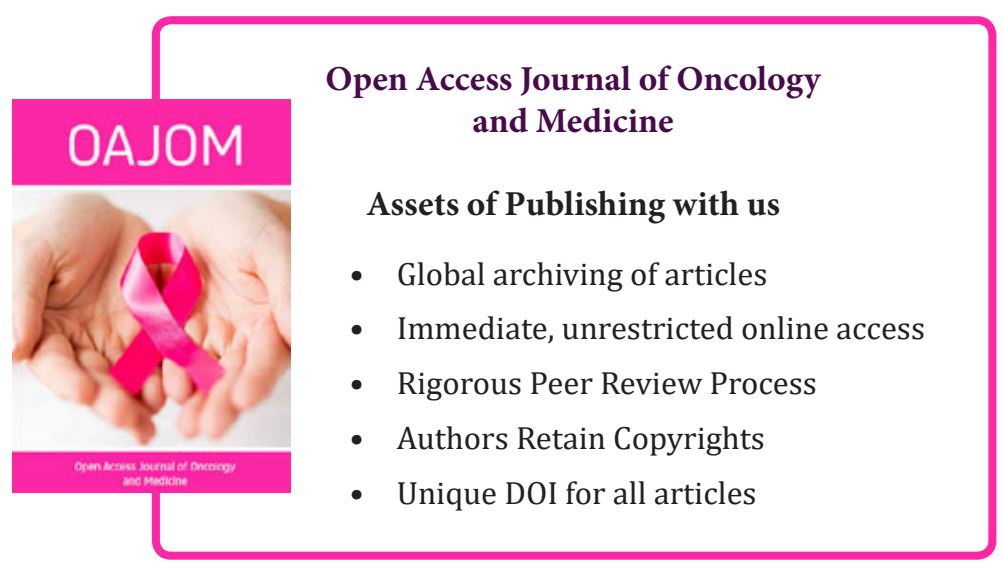

